\section{Cuidados com anticoagulação}

A principal complicação da anticoagulação é o sangramento, mas esse risco não deve ser avaliado isoladamente na decisão do tratamento, sendo importante considerar o potencial benefício da terapia anticoagulante na TEP.(1) $A$ presença de sangramento não deve ser atribuída somente à anticoagulação, sendo indicado investigar a presença de alteração orgânica mesmo quando a anticoagulação encontrar-se em nível terapêutico.(D) São considerados como sangramentos maiores o sangramento intracraniano; o sangramento retroperitoneal, que necessita de hospitalização ou de transfusão de hemoderivados; ou o sangramento fatal.

\section{$A V K$}

Os principais determinantes de sangramento quando em tratamento com AVK são os seguintes: a intensidade e a duração da anticoagulação, o uso de medicações concomitantes e as características do paciente. A idade superior a 75 anos, câncer, hipertensão arterial sistêmica, doença vascular cerebral, doença cardíaca grave, insuficiência renal, doença hepática e alcoolismo também são fatores de risco para o sangramento. Várias medicações podem interferir na farmacocinética da varfarina, aumentando ou diminuindo o seu efeito (Quadro 21). Portanto, é necessário um maior controle da anticoagulação quando qualquer medicação for adicionada ou retirada, inclusive os fitoterápicos. Alimentos ricos em vitamina $\mathrm{K}$, como fígado, vegetais crus e alguns cereais, também podem interferir no controle adequado da anticoagulação com AVK. Se RNl > 4-5 ou se há flutuação no seu controle, ocorre um aumento do risco de sangramento maior. 0 risco de sangramento é maior no primeiro mês, diminuindo nos meses consecutivos no decorrer do primeiro ano; porém, a ocorrência de casos fatais na fase inicial e a longo prazo de tratamento é semelhante. ${ }^{(2-7)} A$ implementação de clínicas de anticoagulação multiprofissional (médicos, farmacêuticos, enfermeiros) tem mostrado melhor eficiência do ajuste terapêutico, tanto em relação a níveis terapêuticos mais frequentes, quanto ao menor indice de sangramentos. ${ }^{(8-10)}$ Recomenda-se, se houver disponibilidade, que os pacientes em uso crônico de anticoagulantes sejam acompanhados em clínicas de anticoagulação.(B)
Outra complicação relacionada à varfarina é a necrose de pele. ${ }^{(11)}$ Essa complicação é rara, mas pode levar a extensas áreas de necrose decorrente da trombose de vênulas e capilares. Geralmente ocorre entre o terceiro e o sexto dia de anticoagulação e está relacionada a doses altas de varfarina no início do tratamento, o que leva a uma rápida redução dos níveis de proteína $\mathrm{C}$ e ocasionando um estado de hipercoagulabilidade transitória.

0 manejo da anticoagulação excessiva dependerá da presença e/ou do risco aumentado de sangramento e do nível de anticoagulação (Quadro 22).(11)

Quadro 21 - Principais interações medicamentosas com os AVK.

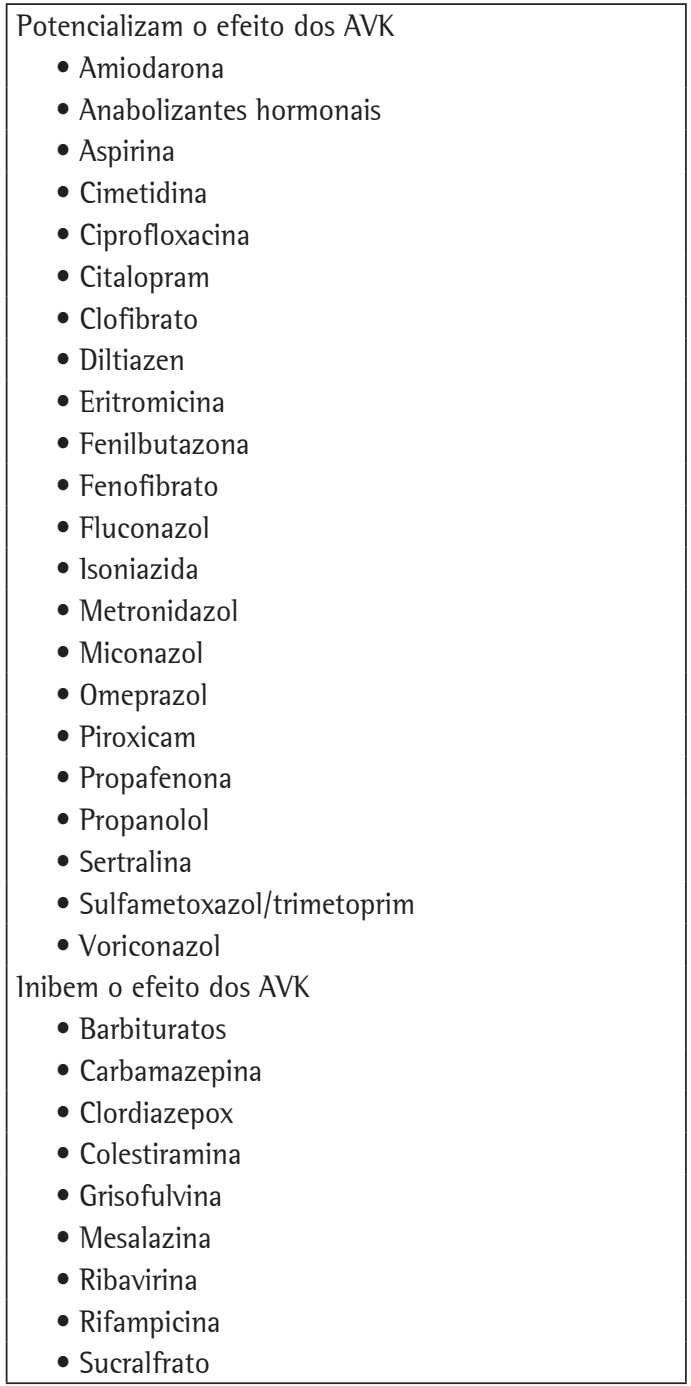

Adaptado do estudo de Ansell et al. ${ }^{(11)}$ 
Quadro 22 - Manejo da elevação de RNI ou da presença de sangramento na vigência de AVK.

\begin{tabular}{|c|c|c|}
\hline $\mathrm{RNI}$ & Sangramento & Conduta \\
\hline$<5$ & Não & $\begin{array}{l}\text { Suspender a próxima dose e/ou reduzir a dose de manutenção. Monitorar com } \\
\text { maior frequência. }\end{array}$ \\
\hline $5-9$ & Não & $\begin{array}{l}\text { Suspender temporariamente ou, além de suspender a varfarina, adicionar } \\
\text { pequena dose de vitamina } \mathrm{K}(<5 \mathrm{mg}) \text { por via oral. Em pacientes com risco de } \\
\text { sangramento ou que necessitarem de reversão mais rápida, repetir vitamina } \mathrm{K} \\
\text { oral }(1-2 \mathrm{mg}) \text {. Reintroduzir a varfarina em dose menor quando se atingir o nível } \\
\text { terapêutico. Monitorar com maior frequência. }\end{array}$ \\
\hline$\geq 9$ & Não & Suspender a varfarina e administrar vitamina $\mathrm{K}$ oral (5 mg). \\
\hline Qualquer nível & Sangramento & $\begin{array}{l}\text { Monitorar a anticoagulação e repetir dose de vitamina K, se necessário. } \\
\text { Reintroduzir a varfarina em dose menor quando se atingir o nível terapêutico. } \\
\text { Suspender a varfarina. }\end{array}$ \\
\hline & grave & $\begin{array}{l}\text { Vitamina K i.v., } 10 \mathrm{mg} \text {, infusão lenta associada a plasma fresco ou concentrado } \\
\text { de complexo protrombínico ou fator Vlla recombinante. }\end{array}$ \\
\hline \multirow[t]{3}{*}{ Qualquer nível } & \multirow{3}{*}{$\begin{array}{l}\text { Sangramento } \\
\text { com risco de } \\
\text { morte }\end{array}$} & Suspender a varfarina. \\
\hline & & $\begin{array}{l}\text { Concentrado de complexo protrombínico ou fator Vlla recombinante. } \\
\text { Vitamina } \mathrm{K} \text { i.v., } 10 \mathrm{mg} \text {, infusão lenta. }\end{array}$ \\
\hline & & Repetir na dependência de RNI. \\
\hline
\end{tabular}

Adaptado do estudo de Ansell et al. ${ }^{(11)}$

A vitamina $\mathrm{K}$ pode ser administrada i.v., mas em infusão lenta. Há risco de reação anafilática. A via s.c. não é recomendada, visto a sua absorção irregular e à baixa eficácia em reverter a anticoagulação.(C) A via intramuscular é contraindicada devido ao risco sangramento local. A via oral é a preferencial, pois a sua eficácia é semelhante à da i.v. mas com menor risco de reação anafilática.(B) A dose oral eficaz é de geralmente 1-2,5 mg. Caso não haja disponibilidade de vitamina $\mathrm{K}$ oral, pode-se administrar o preparado i.v. pela via oral. Deve-se utilizar a medicação i.v. quando houver a necessidade de reversão rápida da anticoagulação.(B) $A$ dose de vitamina $\mathrm{K}$ pode ser repetida a cada $12 \mathrm{~h}$. A reversão do efeito de AVK após a administração da vitamina $\mathrm{K}$ é esperada em 24-48 h. Após doses elevadas de vitamina $\mathrm{K}$, pode haver dificuldades para se atingir a anticoagulação adequada com AVK, recomendando-se a associação de HNF ou HBPM nos primeiros dias. ${ }^{(12)}$

\section{Heparinas}

Os principais efeitos colaterais das heparinas são o sangramento, a trombocitopenia e a osteoporose (sobretudo HNF em doses superiores a $20.000 \mathrm{UI} /$ dia por mais de 30 dias), sendo que os dois últimos são menos frequentes com as HBPM. 0 sangramento pode ocorrer pela inibição dos fatores de coagulação, diminuição da função plaquetária e aumento da permeabili- dade capilar. 0 risco de sangramento com o uso de HNF ou HBPM em níveis terapêuticos é baixo. ldade $>70$ anos, insuficiência renal, cirurgia ou trauma recente, assim como o uso concomitante de ácido acetilsalicílico ou agente trombolítico são fatores de risco para o sangramento associado às heparinas. ${ }^{(13-15)}$

A HNF i.v. intermitentemente traz maior risco de sangramento quando comparada à infusão i.v. contínua, que deve ser o regime preferencial. $(A)^{(16-21)}$

As interações medicamentosas são muito menos frequentes do que com os cumarínicos. Cefalosporinas e penicilinas podem aumentar o risco de sangramento. 0 uso associado de outros antitrombóticos aumenta o risco de hemorragia. Outros fármacos com relato de aumento do efeito da heparina são anti-histamínicos, tetraciclina, quinina, nicotina e digoxina. A nitroglicerina em altas doses pode reduzir o efeito da heparina. ${ }^{(22)}$

0 efeito da heparina é neutralizado com sulfato de protamina, na dose de $1 \mathrm{mg}$ para cada 100 UI de HNF. ${ }^{(23)}$ Para reverter o efeito da HNF em infusão i.v., o cálculo da dose de protamina a ser utilizada deve ser baseado na dose de HNF administrada na última hora.(B) Não existe um método definido para a neutralização do efeito da HBPM. ${ }^{(24)}$ A protamina reverte parcialmente a atividade do antifator Xa da HBPM. Se a HBPM foi administrada nas últimas $8 \mathrm{~h}$, a protamina deve ser dada na dose de $1 \mathrm{mg}$ por $100 \mathrm{UI}$ 
antifator Xa (1 $\mathrm{mg}$ de enoxaparina equivale a aproximadamente $100 \mathrm{UI}$ de antifator $\mathrm{Xa}$ ). Caso o sangramento persista, pode-se repetir a dose de $0,5 \mathrm{mg}$ de protamina para cada $100 \mathrm{Ul}$ de antifator Xa. Quando a HBPM foi administrada a um intervalo maior que $8 \mathrm{~h}$, a dose de protamina necessária é menor.

A protamina é uma proteína derivada de esperma de peixe que se liga à heparina, formando um sal estável. Deve ser administrada lentamente e apresenta risco de reação anafilática. Os pacientes com risco de ter anticorpos antiprotamina (vasectomizados, alergia a peixes e com uso prévio de insulina contendo protamina) devem receber corticoides e anti-histamínicos antes da administração de protamina. ${ }^{(25,26)}$

\section{Referências}

1. Fihn SD, McDonell M, Martin D, Henikoff J, Vermes D, Kent D, et al. Risk factors for complications of chronic anticoagulation. A multicenter study. Warfarin Optimized Outpatient Follow-up Study Group. Ann Intern Med. 1993;118(7):511-20.

2. Landefeld CS, Goldman L. Major bleeding in outpatients treated with warfarin: incidence and prediction by factors known at the start of outpatient therapy. Am J Med. 1989;87(2):144-52.

3. Petitti DB, Strom BL, Melmon KL. Duration of warfarin anticoagulant therapy and the probabilities of recurrent thromboembolism and hemorrhage. Am J Med. 1986;81(2):255-9.

4. Hutten BA, Lensing AW, Kraaijenhagen RA, Prins MH. Safety of treatment with oral anticoagulants in the elderly. A systematic review. Drugs Aging. 1999;14(4):303-12.

5. Lundström T, Rydén L. Haemorrhagic and thromboembolic complications in patients with atrial fibrillation on anticoagulant prophylaxis. J Intern Med. 1989;225(2):137-42.

6. Forfar JC. A 7-year analysis of haemorrhage in patients on long-term anticoagulant treatment. $\mathrm{Br}$ Heart J. 1979;42(2):128-32.

7. Linkins LA, Choi PT, Douketis JD. Clinical impact of bleeding in patients taking oral anticoagulant therapy for venous thromboembolism: a meta-analysis. Ann Intern Med. 2003;139(11):893-900.

8. Chiquette E, Amato MG, Bussey HI. Comparison of an anticoagulation clinic with usual medical care: anticoagulation control, patient outcomes, and health care costs. Arch Intern Med. 1998;158(15):1641-7.

9. Garabedian-Ruffalo SM, Gray DR, Sax MJ, Ruffalo RL. Retrospective evaluation of a pharmacist-managed warfarin anticoagulation clinic. Am J Hosp Pharm. 1985;42(2):304-8.

10. Wilt VM, Gums JG, Ahmed Ol, Moore LM. Outcome analysis of a pharmacist-managed anticoagulation service. Pharmacotherapy. 1995;15(6):732-9.

11. Ansell J, Hirsh J, Hylek E, Jacobson A, Crowther M, Palareti G, et al. Pharmacology and management of the vitamin K antagonists: American College of Chest
Physicians Evidence-Based Clinical Practice Guidelines (8th Edition). Chest. 2008;133(6 Suppl):160S-198S.

12. Dezee KJ, Shimeall WT, Douglas KM, Shumway NM, O'malley PG. Treatment of excessive anticoagulation with phytonadione (vitamin K): a meta-analysis. Arch Intern Med. 2006;166(4):391-7.

13. Mant MJ, O’Brien BD, Thong KL, Hammond GW, Birtwhistle RV, Grace MG. Haemorrhagic complications of heparin therapy. Lancet. 1977;1(8022):1133-5.

14. Jick H, Slone D, Borda IT, Shapiro S. Efficacy and toxicity of heparin in relation to age and sex. $\mathrm{N}$ Engl J Med. 1968;279(6):284-6.

15. Campbell NR, Hull RD, Brant R, Hogan DB, Pineo GF, Raskob GE. Aging and heparin-related bleeding. Arch Intern Med. 1996;156(8):857-60.

16. Glazier RL, Crowell EB. Randomized prospective trial of continuous vs intermittent heparin therapy. JAMA. 1976;236(12):1365-7.

17. Bentley PG, Kakkar WV, Scully MF, MacGregor IR, Webb $\mathrm{P}$, Chan $\mathrm{P}$, et al. An objective study of alternative methods of heparin administration. Thromb Res. 1980;18(1-2):177-87.

18. Andersson G, Fagrell B, Holmgren K, Johnsson H, Ljungberg B, Nilsson E, et al. Subcutaneous administration of heparin. A randomised comparison with intravenous administration of heparin to patients with deep-vein thrombosis. Thromb Res. 1982;27(6):631-9.

19. Doyle DJ, Turpie AG, Hirsh J, Best C, Kinch D, Levine MN, et al. Adjusted subcutaneous heparin or continuous intravenous heparin in patients with acute deep vein thrombosis. A randomized trial. Ann Intern Med. 1987;107(4):441-5.

20. Pini M, Pattachini C, Quintavalla R, Poli T, Megha A, Tagliaferri A, et al. Subcutaneous vs intravenous heparin in the treatment of deep venous thrombosis--a randomized clinical trial. Thromb Haemost. 1990;64(2):222-6.

21. Hull RD, Raskob GE, Hirsh J, Jay RM, Leclerc JR, Geerts $\mathrm{WH}$, et al. Continuous intravenous heparin compared with intermittent subcutaneous heparin in the initial treatment of proximal-vein thrombosis. N Engl J Med. 1986;315(18):1109-14.

22. Amon LC, Gazzana MB. Manejo ambulatorial do paciente anticoagulado. In: Duncan B, Schmidt Ml, Giugliani E, editors. Medicina ambulatorial: condutas de atenção primária baseadas em evidências. Porto Alegre: Artmed; 2005.

23. Hirsh J, Bauer KA, Donati MB, Gould M, Samama MM, Weitz Jl, et al. Parenteral anticoagulants: American College of Chest Physicians Evidence-Based Clinical Practice Guidelines (8th Edition). Chest. 2008;133(6 Suppl):141S-159S.1

24. Institute for Clinical System Improvement. Anticoagulant Therapy Supplement. Bloomington: Institute for Clinical System Improvement; 2001.

25. Protamine Sulfate: Antiheparin agents 20:12.08. In: McEvoy GK, Litvak K, Welsh OH, et al, eds. AHFS drug information 1999. Bethesda MD: American Society of Health-system Pharmacists, 1999; p. 1265-7.

26. Lindblad B, Borgström A, Wakefield TW, Whitehouse WM Jr, Stanley JC. Protamine reversal of anticoagulation achieved with a low molecular weight heparin. The effects on eicosanoids, clotting and complement factors. Thromb Res. 1987;48(1):31-40. 